\title{
Solovay reducibility and continuity
}

\author{
MASAHIRO KUMABE \\ KENSHI MIYABE \\ YUKI MIZUSAWA \\ TOSHIO SUZUKI
}

\begin{abstract}
The objective of this study is a better understanding of the relationships between reducibility and continuity. Solovay reducibility is a variation of Turing reducibility based on the distance of two real numbers. We characterize Solovay reducibility by the existence of a certain real function that is computable (in the sense of computable analysis) and Lipschitz continuous. We ask whether there exists a reducibility concept that corresponds to Hölder continuity. The answer is affirmative. We introduce quasi Solovay reducibility and characterize this new reducibility via Hölder continuity. In addition, we separate it from Solovay reducibility and Turing reducibility and investigate the relationships between complete sets and partial randomness.
\end{abstract}

2010 Mathematics Subject Classification 03D78 (primary); 68Q30 (secondary)

Keywords: Solovay reducibility, Lipschitz continuity, Hölder continuity, computable analysis

\section{Introduction}

We would like to get a better understanding of the relationships between reducibility and continuity.

We say a real number $\alpha$ is left-c.e. if the left cut of $\alpha, L(\alpha):=\{q \in \mathbb{Q} \mid q<\alpha\}$, is computably enumerable. Suppose that $\alpha$ and $\beta$ are left-c.e. real numbers. Observe that there exists a function $f:(-\infty, \beta) \rightarrow(-\infty, \alpha)$ with the following properties.

- $f$ is partially computable in the sense of Weihrauch [15]. (Roughly speaking, the name of $f(x)$ is computable from the name of $x$. We will review the precise definition in Section 2.)

- $\{f(x): x<\beta\}$ is cofinal in $(-\infty, \alpha)$. To be more precise, for any $y<\alpha$ there exists $x<\beta$ such that $y \leq f(x)$. 
- $f$ is nondecreasing.

A sketch of the proof is as follows. Take computable sequences of rationals such that $\left\{a_{n}\right\} \nearrow \alpha$ and $\left\{b_{n}\right\} \nearrow \beta$. Define points $Q_{n}(n \in \mathbb{N})$ on $\mathbb{R}^{2}$ as to be $Q_{n}\left(b_{n}, a_{n}\right)$. We make a line graph by connecting $Q_{n}$ and $Q_{n+1}$ for each $n$. Let $f$ be the function whose graph is the line graph. Then $f$ has the desired properties.

Suppose we add a requirement that $f$ is Lipschitz continuous to the set of properties mentioned above. It is interesting to note that the extended set of properties is exactly equivalent to the assertion that $\alpha$ is Solovay reducible to $\beta$. We show this equivalence in Section 4. Solovay reducibility here is a reducibility between real numbers, and has been deeply studied (Solovay [12], Downey and Hirschfeld [3, Chapter 9]). Solovay reducibility implies Turing reducibility, but the converse implication does not hold. Solovay reducibility has a connection to the theory of randomness. For example, among left-c.e. reals, the completeness with respect to Solovay reducibility agrees with 1-randomness.

We are interested in more examples that show the correspondences between various continuity concepts and reducibility concepts. In the classical analysis, Hölder continuity is one of the well-known continuity concepts.

Definition 1.1 Let $X \subseteq \mathbb{R}$ and $Y \subseteq \mathbb{R}$. A function $f: X \rightarrow Y$ is Hölder continuous if there exist positive real numbers $H$ and $\xi$ such that for any $x_{1}, x_{2} \in X$, the following holds:

$$
\left|f\left(x_{1}\right)-f\left(x_{2}\right)\right|<H\left|x_{1}-x_{2}\right|^{\xi}
$$

where the exponent $\xi$ is called the order.

Throughout the paper, unless otherwise specified, we let Hölder continuity denote that with positive order $\leq 1$. In the case where the domain of the function is a closed interval of the real line, Lipschitz continuity implies Hölder continuity, and Hölder continuity implies uniform continuity.

In the definition of Hölder continuity, the key quantity is the power of the distance of two real numbers. The convergent series of such quantity has been studied as $T$-convergent sequences by Tadaki [14] in the study of partial randomness. Indeed, in this paper, we reveal a strong relationship between Hölder continuity and partial randomness. Another previous work relating to the present paper is the study on partial randomness and Solovay reducibility by Miyabe, Nies and Stephan [10].

Our main question is as follows. Is there a reducibility that exactly corresponds to Hölder continuity? As far as the authors know, there is no previous work that asks this 
question as such. The answer is affirmative. In this paper, we present such a reducibility concept and call it quasi Solovay reducibility.

We give the definitions of our main notion qS-reducibility and several notions in Section 2. In Section 3, we show several properties of qS-reducibility. Especially, we show qS-reducibility is separated from Solovay reducibility and Turing reducibility. In Section 4 , we discuss the relationship of reducibility and continuity.

\section{The background and basic definitions}

\subsection{Computable real numbers and computable real functions}

We let $\mathbb{N}, \mathbb{Q}$, and $\mathbb{R}$ denote the set of all natural numbers, rational numbers, and real numbers, respectively. The set of all binary strings with finite length is denoted by $\{0,1\}^{*}$.

In a suitable definition of computable real functions, the usual Turing machine would be insufficient as a model of computation. A number of previous studies have prospected suitable definitions. Among them, Ko and Friedman [7] introduced a definition based on an oracle Turing machine. In this approach, roughly speaking, computability of a real function $f$ is defined in the following way. An independent real variable $x$ is considered as an oracle function; in other words, an idealized library function. We consider the mapping of $0^{n}=0 \cdots 0$ ( $n$ times, where $n$ is a natural number) to a rational number $q$ approximating $f(x)$ with error at most $2^{-n}$. In general, we do not require computability of $x$. We require that for each $x$ in the domain of $f$, the above-mentioned mapping of $0^{n}$ to $q$ is computable using $x$ as an oracle, where the algorithm is uniform in the sense that it depends only on $f$ and is independent of $x$.

A variation of the Ko-Friedman style definition is precisely given in the textbook by Weihrauch [15]. An equivalent definition is given in Kawamura, Thies and Ziegler [6].

Definition 2.1 (Grzegorczyk [4], Ko and Friedman [7] and Kawamura, Thies and Ziegler [6]) Suppose that $x$ is a real number.

(1) A name of $x$ is a function $\varphi:\{0,1\}^{*} \rightarrow\{0,1\}^{*}$ such that for each string $u$, letting $n$ be the length of $u, \varphi(u)$ is a binary encoding of an integer $z$ such that $\left|x-z / 2^{n}\right| \leq 2^{-n}$.

(2) $x$ is a computable real number if it has a computable name. 
Suppose that $x$ is a real number with $0 \leq x \leq 1$. It is well-known that the following (i) and (ii) hold. (i) Any real number $x$ has infinitely many names. (ii) $x$ is computable in the above-mentioned sense if and only if there is a total computable function $f: \mathbb{N} \rightarrow\{0,1\}$ such that $0 . f(0) f(1) f(2) \cdots$ is a binary expansion of $x$.

Several types of oracle Turing machines have been discussed in the literature. Here, we consider an oracle Turing machine with a function oracle. An oracle Turing machine is a Turing machine equipped with a particular read-write tape called an oracle tape, and the particular state $q_{\text {query }}$ called the query state. Suppose that $f$ is a mapping from strings to strings. An oracle Turing machine $M$ with an oracle $f$, denoted by $M^{f}$, is similar to the usual Turing machine that computes a function. It gets an input string from a certain tape, and it outputs a string on a certain tape. However, the action of $M^{f}$ differs from those of the usual machine in the following points. When $M$ enters the query state, $M$ writes a string, say $u$, on the oracle tape. The action of writing is regarded as $|u|$ time-steps, where $|u|$ is the length of $u$. Then $u$ is replaced by the string $f(u)$, and $M$ enters another state. This replacement is regarded as one time-step. When $M$ reads $f(u)$, we count time-steps in the same way as we have done at the writing action.

An outline of the definition of a computable real function in Weihrauch [15] is as follows. This would be sufficient for our purpose. For more rigorous treatment, consult [15].

Definition 2.2 (Kawamura, Thies and Ziegler [6]) Suppose that $f$ is a partial function from $\mathbb{R}$ to $\mathbb{R}$. The function $f$ is computable if there exists an oracle Turing machine $M$ with the following property. For each $x$ in the domain of $f$ and for each name $\varphi$ of $x$, $M^{\varphi}$ computes a name of $f(x)$.

By saying that a real function is partially computable in the sense of Weihrauch, we mean the above-mentioned sense. We will consider the case where such a partial computable function is defined on an open interval $(-\infty, \beta)$.

\subsection{Left-c.e. real numbers and reducibilities}

Unless otherwise specified, $\left\{a_{n}\right\} \nearrow \alpha$ denotes that $\left\{a_{n}\right\}$ is a computable nondecreasing sequence of rationals converging to $\alpha$. For a function $f$, the symbol $f(x) \downarrow$ denotes that $f$ is defined on $x$. For a Turing machine $M$, the symbol $M(x) \downarrow$ denotes that $M$ terminates in a finite step for input $x$. A set $A$ of natural numbers is computably enumerable, c.e. for short, if there exists a Turing machine $M$ such that $A=\{n \in \mathbb{N}: M(n) \downarrow\}$. The 
concept of c.e. sets is naturally generalized to a set of rational numbers, or to a set of binary strings, under a certain coding.

Although the concept of computable real numbers is quite natural, the class of all computable real numbers is narrow in the field of algorithmic randomness, for any computable real number is not random. A fruitful relaxed class is the class of all left-c.e. real numbers. As we defined in the introduction section, a real number is left-c.e. if the left cut is computably enumerable. A typical example of a left-c.e. random real number is Chaitin's $\Omega$. For details on Chaitin's $\Omega$ and related concepts, consult standard textbooks of algorithmic randomness such as Downey and Hirschfeld [3] and Nies [11].

Turing reducibility is a concept that compares the complexity of two functions $f, g: \mathbb{N} \rightarrow$ $\{0,1\} . f$ is Turing reducible to $g$ ( $f \leq_{T} g$ in symbol) if there exists an oracle Turing machine $M$ such that for each natural number $n, M^{g}$ halts for input $n$ and outputs $f(n)$. Turing reduction between two sets of natural numbers means Turing reduction between their characteristic functions. For real numbers $\alpha$ and $\beta$, let $0 . f(1) f(2) \cdots$ and $0 . g(1) g(2) \cdots$ be their binary expansions such that in each expansion, 0 has infinitely many occurrences. It is easy to see that $f$ is Turing reducible to $g$ if and only if the left cut of $\alpha$ is Turing reducible to the left cut of $\beta$. Turing reduction between two real numbers is defined with this meaning, that is, Turing reduction between their left cuts. If $\alpha \leq_{T} \beta$, we also say $\alpha$ is $\beta$-computable.

In the computability theory, quite a few variations of Turing reducibility have been introduced. Interestingly enough, many important reducibility concepts have common properties that are known as standard in the following sense.

Definition 2.3 [3, Chapter 9] The reducibility $r$ is standard if the following hold.

(1) $r$ is $\Sigma_{3}^{0}$.

(2) Every computable real is reducible to any given left-c.e.real.

(3) Real addition is the join in the r-degrees of left-c.e.reals.

(4) For any left-c.e. real $\alpha$ and any rational $q>0$, we have $\alpha \equiv_{r} q \alpha$.

The condition (3) of the above definition means that, if we restrict ourselves to the left-c.e. reals, the least upper bound of the r-degrees of real $\alpha$ and the r-degree of real $\beta$ is given by the r-degree of real $\alpha+\beta$.

Turing reducibility is, of course, a standard reducibility. Another important example of a standard reducibility is Solovay reducibility. Suppose that $\alpha$ and $\beta$ are real numbers. $\alpha \leq_{S} \beta$ ( $\alpha$ is Solovay reducible to $\beta$ ) [3, Chapter 9] if there exist a partial computable 
function $f$ from $\mathbb{Q}$ to $\mathbb{Q}$ and a positive natural number $d$ with the following property. For any rational $x<\beta$, we have $f(x) \downarrow<\alpha$ and $\alpha-f(x)<d(\beta-x)$. The following is the main notion of this paper, which is a slight modification of Solovay reducibility.

Definition 2.4 $\alpha \leq_{q S} \beta$ ( $\alpha$ is quasi Solovay reducible to $\beta$ ) if there exist a partial computable function $f$ from $\mathbb{Q}$ to $\mathbb{Q}$ and positive natural numbers $d, \ell$ with the following property. For any rational $x<\beta$, we have $f(x) \downarrow<\alpha$ and $(\alpha-f(x))^{\ell}<d(\beta-x)$ (in other words, $(\alpha-f(x))<H(\beta-x)^{1 / \ell}$, where $\left.H=d^{1 / \ell}\right)$.

\section{The relationships among the reducibilities}

There are some known characterizations of Solovay reducibility by means of sequences of rational numbers. A characterization by Calude, Coles, Hertling and Khoussainov [2] (see also [3, Proposition 9.12]) can be generalized to quasi Solovay reducibility as follows.

Lemma 3.1 Suppose that $\alpha$ and $\beta$ are left-c.e. reals. Then the following are equivalent.

(1) $\alpha \leq_{q S} \beta$.

(2) For every $\left\{a_{n}\right\} \nearrow \alpha$ and $\left\{b_{n}\right\} \nearrow \beta$, there exist an increasing computable function $g: \mathbb{N} \rightarrow \mathbb{N}$ and positive integers $d$ and $\ell$ such that for each $n \in \mathbb{N}$, the following holds.

$$
\left(\alpha-a_{g(n)}\right)^{\ell} \leq d\left(\beta-b_{n}\right)
$$

(3) For every $\left\{b_{n}\right\} \nearrow \beta$, there exist $\left\{a_{n}\right\} \nearrow \alpha$ and positive integers $d$ and $\ell$ such that for each $n \in \mathbb{N}$, the following holds.

$$
\left(\alpha-a_{n}\right)^{\ell} \leq d\left(\beta-b_{n}\right)
$$

(4) There exist $\left\{a_{n}\right\} \nearrow \alpha,\left\{b_{n}\right\} \nearrow \beta$ and positive integers $d$ and $\ell$ such that for each $n \in \mathbb{N}$, the following holds.

$$
\left(\alpha-a_{n}\right)^{\ell} \leq d\left(\beta-b_{n}\right)
$$

Proof Proof of $(1) \Rightarrow(2)$ : Let $f: \mathbb{Q} \rightarrow \mathbb{Q}, d$ and $\ell$ be witnesses of $\alpha \leq_{q S} \beta$. We are going to define a mapping $g: \mathbb{N} \rightarrow \mathbb{N}$ by means of recursion. Given $n \in \mathbb{N}$, find the least $s \in \mathbb{N}$ such that $f\left(b_{n}\right)<a_{s}$ and $s>g(i)$ (for all $i<n$ ). Then we define $g(n)$ as to be this $s$. 
Proof of $(2) \Rightarrow(3)$ : Since $\alpha$ is left-c.e., there exists a sequence $\left\{a_{n}\right\} \nearrow \alpha$. Take a $g: \mathbb{N} \rightarrow \mathbb{N}$ in the statement of assertion 2. The sequence $\left\{a_{g(n)}\right\}$ is what we want.

(3) $\Rightarrow(4)$ is obvious.

Proof of (4) $\Rightarrow(1)$ : Let $\left\{a_{n}\right\} \nearrow \alpha,\left\{b_{n}\right\} \nearrow \beta, d$ and $\ell$ be witnesses of assertion 4. For each rational number $q<\beta$, find a natural number $n$ such that $b_{n} \leq q<b_{n+1}$. Define $f(q)$ as to be $a_{n+1}$. Then it holds that $(\alpha-f(q))^{\ell}<d(\beta-q)$.

Lemma 3.2 Let $\leq_{q S}$ be the relation given by quasi Solovay reduction on the left-c.e. reals.

(1) $\leq_{q S}$ is a pre-ordering.

(2) $\leq_{q S}$ is a standard reducibility in the sense of Definition 2.3.

Proof (1) (Reflexivity) For each real $\alpha$, it holds that $|\alpha-x|^{1}<2|\alpha-x|$. Thus we have $\alpha \leq_{q S} \alpha$.

(Transitivity) Suppose that $\alpha \leq_{q S} \beta$ holds with witness $\ell_{1}, d_{1}, f$ and that $\beta \leq_{q S} \gamma$ holds with witness $\ell_{2}, d_{2}, g$. We define $h=f \circ g$. For each rational $q<\gamma, g(q) \downarrow<\beta$ and $h(q)=f \circ g(q) \downarrow<\alpha$.

Now we have $(\alpha-h(q))^{\ell_{1}}<d_{1}(\beta-g(q))$ and $(\beta-g(q))^{\ell_{2}}<d_{2}(\gamma-q)$ for each rational $q$. So we have $(\alpha-h(q))^{\ell_{1} \ell_{2}}<d_{1}^{\ell_{2}}(\beta-g(q))^{\ell_{2}}<d_{1}^{\ell_{2}} d_{2}(\gamma-q)$ for each rational $q<\gamma$.

(2) The conditions (1), (2) and (4) of Definition 2.3 hold for $\leq_{q S}$ because $\leq_{S}$ is a standard reducibility.We prove the following proposition for (3) of Definition 2.3.

Claim: Suppose that $\alpha$ and $\beta$ are left-c.e. reals. Then we have the following.

$$
\operatorname{deg}_{q S}(\alpha+\beta)=\sup \left\{\operatorname{deg}_{q S} \alpha, \operatorname{deg}_{q S} \beta\right\}
$$

Here, the supremum is taken among left-c.e. reals.

To prove the claim, it suffices to show the following two propositions:

(i) $\alpha \leq_{q S} \alpha+\beta$ and $\beta \leq_{q S} \alpha+\beta$.

(ii) For a left-c.e.real $\gamma$, if $\alpha \leq_{q S} \gamma$ and $\beta \leq_{q S} \gamma$ then $\alpha+\beta \leq_{q S} \gamma$.

Proof of (i): We have $\alpha \leq_{S} \alpha+\beta$ by Definition 2.4 and $\leq_{S}$ implies $\leq_{q S}$. Hence $\alpha \leq_{q S} \alpha+\beta$. We have $\beta \leq_{q S} \alpha+\beta$ by the same argument as above.

Proof of (ii): Suppose that $\gamma$ is a left-c.e.real and we have $\alpha \leq_{q S} \gamma$ and $\beta \leq_{q S} \gamma$. Let $\left\langle f_{0}, c_{0}, \ell_{0}\right\rangle$ and $\left\langle f_{1}, c_{1}, \ell_{1}\right\rangle$ be witnesses, respectively. If $q \in \mathbb{Q}$ and $q<\gamma$ then 
$f_{0}(q) \downarrow<\alpha,\left(\alpha-f_{0}(q)\right)^{\ell_{0}}<c_{0}(\gamma-q)$ and $f_{1}(q) \downarrow<\beta,\left(\beta-f_{1}(q)\right)^{\ell_{1}}<c_{1}(\gamma-q)$. We can assume $\ell_{0}<\ell_{1}, c_{0} \geq 1, c_{1} \geq 1$ and $\gamma-q<1$ without loss of generality. We set $f_{2}(x)=f_{0}(x)+f_{1}(x)$. Then it holds that $f_{2}(q) \downarrow<\alpha+\beta$, and we have the following.

$$
\begin{aligned}
(\alpha+\beta)-f_{2}(q) & <c_{0}^{1 / \ell_{0}}(\gamma-q)^{1 / \ell_{0}}+c_{1}^{1 / \ell_{1}}(\gamma-q)^{1 / \ell_{1}} \\
& \leq c_{0}^{1 / \ell_{0}}(\gamma-q)^{1 / \ell_{1}}+c_{1}^{1 / \ell_{0}}(\gamma-q)^{1 / \ell_{1}} \\
& =\left(c_{0}^{1 / \ell_{0}}+c_{1}^{1 / \ell_{0}}\right)(\gamma-q)^{1 / \ell_{1}}
\end{aligned}
$$

We set $c_{2}:=\left(c_{0}^{1 / \ell_{0}}+c_{1}^{1 / \ell_{0}}\right)^{\ell_{1}}$ then $\left((\alpha+\beta)-f_{2}(q)\right)^{\ell_{1}} \leq c_{2}(\gamma-q)$. Therefore $\alpha+\beta \leq_{q S} \gamma$ via $f_{2}, c_{2}, \ell_{1}$.

A real number in the unit interval is, by taking its binary expansion in which 0 has infinitely many occurrences, often identified with an infinite binary sequence. In this case, we need to be aware of the following point. Suppose that we know that $|\beta-\alpha|$, the geometrical distance of two real numbers $\alpha$ and $\beta$ in the real line, is at most $2^{-n}$. In general, this assumption does not imply the agreement between the first $n$ bits of $\alpha$ and those of $\beta$ (their binary expansions in the above-mentioned style). For example, observe the case where $\alpha=0.00111110, \beta=0.010$ and $n=7$. This delicate relationship between geometrical distance and agreement of bits sometimes appears as an obstacle in the study of Solovay reducibility. In order to avoid such an obstacle, we introduce a set $R^{\prime}$ of numbers, as follows.

Recall that a dyadic rational is a rational number of the form $q=z 2^{-n}$ where $z \in \mathbb{Z}$ and $n \in \mathbb{N}$ (Nies [11, Chapter 1]). Now we look at a dyadic rational $q$ with the following property.

(3-6) $q$ has a binary expansion $q=0 . q_{1} q_{2} \cdots q_{2 k-1} q_{2 k}$,

$$
\text { where for each } i, \quad q_{2 i}=1-q_{2 i-1} \text {. }
$$

When it is apparent from the context that $q$ is a dyadic rational satisfying (3-6), we let $k(q)$ denote the above-mentioned $k$. For example, 0.1010 has property (3-6) with $k(0.1010)=2$. On the other hand, 0.1110 does not have property (3-6).

Definition 3.3 $R^{\prime}$ is the set of all reals $\beta$ with the following properties.

(1) $\beta$ is not a rational.

(2) $\beta$ has a binary expansion $\beta=0 . b_{1} b_{2} \cdots$ with the following property.

$$
\forall n \in \mathbb{N}^{+}\left[0 . b_{1} b_{2} \cdots b_{2 n-1} b_{2 n}\right. \text { satisfies (3-6).] }
$$

Lemma 3.4 Suppose that $\alpha$ and $\beta$ are left-c.e. reals. 
(1) $\alpha \leq_{S} \beta$ implies $\alpha \leq_{q S} \beta$.

(2) $\alpha \leq_{q S} \beta$ does not imply $\alpha \leq_{S} \beta$.

Proof (1) follows by setting $\ell$ in the definition to be 1 .

(2) Claim 1: Suppose that $q$ is a rational satisfying (3-6), $\beta \in R^{\prime}, q<\beta$ and that $|\beta-q| \leq 2^{-(2 m+1)}$ for some $m \in \mathbb{N}$. Then, the following holds.

$$
\forall i \in \mathbb{N}\left[1 \leq i \leq 2 m \Rightarrow b_{i}=q_{i}\right]
$$

Proof of Claim 1: We prove this claim by induction. Base step $m=0$ : the claim is obvious.

Induction step $m=s+1$ : Our induction hypothesis is as follows.

$$
1 \leq i \leq 2 s \Rightarrow b_{i}=q_{i}
$$

Case 1: $b_{2 s+1} b_{2 s+2}=01$.

If $q_{2 s+1} q_{2 s+2}=10$ then $\beta<0 . b_{1} \cdots b_{2 s} 0111$ and $q \geq 0 . b_{1} \cdots b_{2 s} 10$. Hence $q>\beta$. We have a contradiction.

If $q_{2 s+1} q_{2 s+2}=00$ (which may well happen in the case of $k(q)<s$ ) then $\beta>$ $0 . b_{1} \cdots b_{2 s} 0101$ and $q=0 . b_{1} \cdots b_{2 s}$. Hence:

$$
\beta-q>0 . \underbrace{0 \cdots 0}_{2 s} 0101>0 . \underbrace{0 \cdots 0}_{2 s} 001=2^{-(2 m+1)}
$$

We have a contradiction. Hence $q_{2 s+1} q_{2 s+2}=01$ holds. Therefore the following holds.

$$
1 \leq i \leq 2 s+2 \Rightarrow b_{i}=q_{i}
$$

Case 2: Otherwise. In other words $b_{2 s+1} b_{2 s+2}=10$.

If $q_{2 s+1} q_{2 s+2}=01$ then $\beta>0 . b_{1} \cdots b_{2 s} 1001$ and $q \leq 0 . b_{1} \cdots b_{2 s} 0111$. Hence:

$$
\beta-q>0 . \underbrace{0 \cdots 0}_{2 s} 001=2^{-(2 m+1)}
$$

We have a contradiction.

If $q_{2 s+1} q_{2 s+2}=00$ we can derive a contradiction by the same argument as above. Hence $q_{2 s+1} q_{2 s+2}=10$ holds. Therefore the following holds.

$$
1 \leq i \leq 2 s+2 \Rightarrow b_{i}=q_{i}
$$

Claim 1 is proved. 
We are going to show the existence of left-c.e.reals $\alpha$ and $\beta$ such that $\alpha \not_{S} \beta$ and $\alpha \leq_{q S} \beta$. Given $\alpha$, let $0 . \alpha(0) \alpha(1) \cdots$ be its binary expansion that has infinitely many occurrences of 0 . Thus $\alpha(n)$ is the $n+1$ st decimal place. For each $n \in \mathbb{N}$, we define:

$$
h_{1}(\alpha)(2 n):=\alpha(n) \text { and } h_{1}(\alpha)(2 n+1):=1-\alpha(n)
$$

\section{Claim 2:}

(1) $\alpha$ is left-c.e. $\Rightarrow h_{1}(\alpha)$ is left-c.e.

(2) $\alpha$ and $h_{1}(\alpha)$ are left-c.e. $\Rightarrow \alpha \leq_{q S} h_{1}(\alpha)$.

Proof of (1) of Claim 2: If $\alpha$ is a left-c.e.real then there exists a computable increasing sequence of rationals $\left\{a_{n}\right\}_{n \in \mathbb{N}}$ converging to $\alpha$. We can assume that $a_{n}$ is a dyadic rational for all $n \in \mathbb{N}$.Then $a_{n}=\sum_{i=1}^{k} a_{i}^{(n)} 2^{-i}\left(a_{i}^{(n)}=0\right.$ or 1$)$ for some $k$. We define a computable sequence of rationals $\left\{b_{n}\right\}_{n \in \mathbb{N}}$ such that $b_{n}=\sum_{i=1}^{k}\left(a_{i}^{(n)}+1\right) 4^{-i}$ for each $n \in \mathbb{N}$. The sequence is increasing and converges to $h_{1}(\alpha)$.

Proof of (2) of Claim 2: Let $\alpha$ be a left-c.e.real and $\beta=h_{1}(\alpha)$. We are going to prove that there exists a partial computable function $f$ with the following property.

$$
\forall q \in \mathbb{Q}\left[\beta-2^{-5}<q<\beta \Rightarrow\left(f(q) \downarrow<\alpha \wedge(\alpha-f(q))^{4}<\beta-q\right)\right]
$$

Definition of $f$ : Given rational number $q<\beta$, we can effectively find a dyadic rational $q^{\prime}$ such that $q \leq q^{\prime}<\beta$ and $q^{\prime}$ satisfies (3-6) because $\beta=h_{1}(x)$ is in $R^{\prime}$. For $q^{\prime}=0 . q_{1}^{\prime} q_{2}^{\prime} \cdots q_{2 k-1}^{\prime} q_{2 k}^{\prime}(k=k(q))$, we define $f(q):=0 . q_{1}^{\prime} q_{3}^{\prime} \cdots q_{2 k-1}^{\prime}$.

Verification: Case 1 . If there exists $m \in \mathbb{N}$ such that $m \geq 2$ and $2^{-(2 m+2)}<\beta-q^{\prime} \leq$ $2^{-(2 m+1)}$ then by Claim 1, $\alpha$ and $f(q)$ have binary expansions whose first $m$ bits coincide. Hence $|\alpha-f(q)| \leq 2^{-m}$ and $|\alpha-f(q)|^{4} \leq 2^{-4 m} \leq 2^{-(2 m+2)}<\left|\beta-q^{\prime}\right| \leq|\beta-q|$. In other words, $(\alpha-f(q))^{4}<\beta-q$.

Case 2. Otherwise. There exists $m \in \mathbb{N}$ such that $m \geq 2$ and $2^{-(2 m+3)}<\beta-q^{\prime} \leq$ $2^{-(2 m+2)}$. Then by Claim $1, \alpha$ and $f(q)$ have binary expansions whose first $m$ bits coincide. Hence $|\alpha-f(q)| \leq 2^{-m}$ and $|\alpha-f(q)|^{4} \leq 2^{-4 m} \leq 2^{-(2 m+3)}<\left|\beta-q^{\prime}\right| \leq$ $|\beta-q|$. In other words, $(\alpha-f(q))^{4}<\beta-q$. Therefore $\alpha \leq_{q S} \beta$. Claim 2 is proved.

Let $\alpha=\Omega$ and $\beta=h_{1}(\alpha) . \alpha \not_{S} \beta$ because $\alpha$ is 1-random and $\beta$ is not 1-random (the Kolmogorov complexities of $\beta$ are small). Therefore we have left-c.e.reals $\alpha$ and $\beta$ such that $\alpha \not_{S} \beta$ and $\alpha \leq_{q S} \beta$.

Lemma 3.5 Suppose that $\alpha$ and $\beta$ are left-c.e. reals.

(1) $\alpha \leq_{q S} \beta$ implies $\alpha \leq_{T} \beta$. 
(2) $\alpha \leq_{T} \beta$ does not imply $\alpha \leq_{q S} \beta$.

Proof (1) Suppose that $\langle f, d, \ell\rangle$ is a witness of the qS-reducibility. Take a $\beta$-computable sequence $\left\{\gamma_{n}\right\} \nearrow \beta$ of the following property.

$$
\forall n \beta-\gamma_{n} \leq 2^{-\ell n}
$$

Then we have the following.

$$
\alpha-f\left(\gamma_{n}\right) \leq d^{1 / \ell}\left(\beta-\gamma_{n}\right)^{1 / \ell} \leq d^{1 / \ell} 2^{-n}
$$

Hence, $\alpha$ is $\beta$-computable.

(2) We are going to show the existence of reals $\alpha$ and $\beta$ such that $\alpha \leq_{T} \beta$ and $\alpha \not_{q S} \beta$. Let $\Omega=0 . \alpha_{1} \alpha_{2} \alpha_{3} \cdots \alpha_{n} \cdots$ be the binary expansion of Chaitin's halting probability $\Omega$. Let $\beta$ be the real number whose binary expansion is given as follows.

$$
\beta=0 . \alpha_{1} \alpha_{2} \alpha_{2} \alpha_{3} \alpha_{3} \alpha_{3} \cdots \underbrace{\alpha_{n} \cdots \alpha_{n}}_{n} \cdots
$$

Then $\Omega \equiv_{T} \beta$. Assume for a contradiction that $\Omega \leq_{q S} \beta$. Then there exist $\ell, k \in \mathbb{N}$ and a partial computable function $f: \mathbb{Q} \rightarrow \mathbb{Q}$ such that for all rationals $q<\beta, f(q)$ is defined and $(\Omega-f(q))^{\ell}<2^{k}(\beta-q)$. For each bit string $\sigma=x_{1} \cdots x_{m}$, we define the rational $q(\sigma)$ as

$$
q(\sigma)=0 . x_{1} x_{2} x_{2} x_{3} x_{3} x_{3} \cdots \underbrace{x_{m} \cdots x_{m}}_{m}
$$

In particular, in the case where $\sigma$ is the first $m$ bits $\alpha_{1} \cdots \alpha_{m}$ of $\Omega, q=q(\sigma)$ is the first $m(m+1) / 2$ bits of $\beta$. In this case we have $(\Omega-f(q))^{\ell}<2^{k}(\beta-q)<2^{k-m(m+1) / 2}$, thus $\Omega-f(q)<2^{-\left(m^{2}-2 k\right) /(2 \ell)}$. Therefore $\Omega$ belongs to the following interval:

$$
I_{\sigma}=\left(f(q)-2^{-\left(m^{2}-2 k\right) /(2 \ell)}, \quad f(q)+2^{-\left(m^{2}-2 k\right) /(2 \ell)}\right)
$$

By means of these intervals, we construct a Martin-Löf test. We define $U_{m}$ as to be the union of all $I_{\sigma}$ among all binary strings $\sigma$ of length $m$ such that $f(q(\sigma))$ is defined. Then $\left\{U_{m}\right\}_{m}$ is uniformly $\Sigma_{1}$. In addition, $\lambda\left(U_{m}\right) \leq 2^{m} \cdot 2 \cdot 2^{-\left(m^{2}-2 k\right) /(2 \ell)}$. Thus for all but finitely many $m$, it holds that $\lambda\left(U_{m}\right) \leq 2^{-m}$. By the previous paragraph, $\Omega$ belongs to $U_{m}$ for all $m$. This contradicts the fact that $\Omega$ is Martin-Löf random.

Now, we are going to investigate the relation to partial randomness. For an infinite binary sequence $\alpha=a_{0} a_{1} a_{2} a_{3} \cdots$ that is Martin-Löf random, $\beta=a_{0} 0 a_{1} 0 a_{2} 0 a_{3} \ldots$ is not Martin-Löf random. However, it is natural to regard $\beta$ as a partial random sequence. Some important investigations in the earlier stage of partial randomness are 
in Tadaki [13]. See Downey and Hirschfeld [3, Chapter 13] or Lutz [8] for a summary of partial randomness, also known as effective dimension. Some generalizations of partial randomness concepts have been discussed in Higuchi, Hudelson, Simpson and Yokoyama [5]. Here, we review the following terminology from [13].

Definition 3.6 We use the symbol $\leq^{+}$in such a way that $f(n) \leq^{+} g(n)$ denotes that for some constant $c$ it holds that $f(n) \leq g(n)+c$. Let $T \in(0,1]$ be a real number. A real number $\alpha$ is weakly Chaitin $T$-random if:

$$
\forall n \in \mathbb{N}^{+}\left[T n \leq^{+} K\left(\alpha \uparrow_{n}\right)\right]
$$

Definition 3.7 Let $T \in(0,1]$ be a real number. A real number $\alpha$ is $T$-compressible if:

$$
K\left(\alpha \uparrow_{n}\right) \leq T n+o(n)
$$

The following lemma illustrates that quasi Solovay reducibility induces an upper bound on prefix-free Kolmogorov complexity.

Lemma 3.8 Suppose that $\alpha$ and $\beta$ are left-c.e.reals. If $\alpha \leq_{q S} \beta$ with witness $\langle f, d, \ell\rangle$, where $\beta \uparrow_{(\ell \times n)}=\sigma_{1} \frown \cdots \frown \sigma_{\ell},\left|\sigma_{i}\right|=n$ then the following holds.

$$
K\left(\alpha \uparrow_{n}\right) / \ell \leq \max _{i}\left\{K\left(\sigma_{i}\right)\right\}+O(1)
$$

Proof We can compute $\alpha \uparrow_{n}$ from $\beta \uparrow_{(\ell \times n)}$ and little more constant bits. To be more precise, letting $q=\beta \uparrow_{(\ell \times n)}$, we have $0<(\alpha-f(q))^{\ell} \leq d(\beta-q) \leq d 2^{-(\ell \times n)}$. Therefore we have $0<\alpha-f(q)<d^{1 / \ell} 2^{-n}$. Let $m$ be the least positive integer $\geq 1+d^{1 / \ell}$. Then $0<\mid \alpha\left\lceil_{n}-f(q) \mid<m 2^{-n}\right.$. There are at most $2 m$ many possible values of $\alpha \uparrow_{n}$. Letting $t \in\{1, \ldots, 2 m\}$ be such that $\alpha \uparrow_{n}$ is the $t$ th from the least among the possible values, $\alpha \uparrow_{n}$ is determined by $\beta \uparrow_{(\ell \times n)}$ and $t \leq 2 m$. Therefore, the following hold.

$$
\begin{aligned}
K\left(\alpha \uparrow_{n}\right) & \leq K\left(\beta \uparrow_{(\ell \times n)}\right)+O(1) \\
& =K\left(\sigma_{1} \frown \cdots \frown \sigma_{\ell}\right)+O(1) \\
& \leq K\left(\sigma_{1}\right)+\cdots+K\left(\sigma_{\ell}\right)+O(1) \\
& \leq \ell \max _{i}\left\{K\left(\sigma_{i}\right)\right\}+O(1)
\end{aligned}
$$

The lemma has been proved.

In the study of partial randomness, Tadaki [13] introduced the concept of generalized halting probability $\Omega^{T}$ for each positive real number $T \leq 1$.

$$
\Omega^{T}:=\sum_{p \in \operatorname{dom} U} 2^{-|p| / T}
$$


Here, $U$ is a fixed universal prefix-free machine. In the case of $T=1, \Omega^{1}$ coincides with the usual Chaitin's halting probability $\Omega$. In particular, Tadaki showed that if $0<T \leq 1$ and $T$ is computable then $\Omega^{T}$ is weakly Chaitin $T$-random and $T$-compressible [13]. The weakly Chaitin $T$-randomness has been shown in Mayordomo [9] too.

In the case of $T=2^{-n}$ and $n$ is a natural number, we introduce a modified generalized halting probability $\Omega_{T}$. Recall that we defined function $h_{1}$ so that $h_{1}(\alpha)(2 n)=\alpha(n)$ and $h_{1}(\alpha)(2 n+1)=1-\alpha(n)$ (in the proof of Lemma $3.4(2)$ ).

$$
\Omega_{2^{0}}:=\Omega, \quad \Omega_{2^{-(n+1)}}:=h_{1}\left(\Omega_{2^{-n}}\right)
$$

Lemma 3.9 Let $n \in \mathbb{N}$ and $T=2^{-n}$.

(1) $\Omega_{T}$ is a left-c.e. real number.

(2) $\Omega_{T}$ is $q S$-complete among left-c.e.reals.

(3) $\Omega_{T}$ is weakly Chaitin $T$-random.

(4) $\Omega_{T}$ is $T$-compressible.

Proof The case of $n=0$ is a well-known fact. We are going to prove the case of $n=1$. The assertion (1) is immediate from Claim 2 (1) in the proof of Lemma 3.4 (2) and the fact that $\Omega$ is left-c.e. The assertion (2) holds because $\Omega$ is S-complete and $\Omega \leq_{q S} h_{1}(\Omega)$ by Claim 2 (2). For all $k \in \mathbb{N}$, we have $K\left(\Omega \uparrow_{k}\right) \leq^{+} K\left(h_{1}(\Omega) \uparrow_{2 k}\right) \leq^{+} K\left(\Omega \uparrow_{k}\right)$. Hence $k \leq^{+} K\left(h_{1}(\Omega)\left\lceil_{2 k}\right) \leq^{+} k+2 \log k\right.$ (see [11, Section2.2]). If $k$ is even then $k / 2 \leq^{+} K\left(h_{1}(\Omega) \uparrow_{k}\right) \leq^{+} k / 2+2 \log k / 2=k / 2+o(k)$. If $k$ is odd then the complexity differs from the even-case at most up to a constant. Therefore the assertions (3) and (4) hold for $n=1$. The induction step is shown in the same way as the above-mentioned case of $n=1$. Thus, the assertions hold for all $n \in \mathbb{N}$.

\section{The reducibilities and notions of continuity}

In the former half of this section, we show that the existence of a Lipschitz continuous function characterizes Solovay reducibility. In the latter half of this section, we extend the method of the former half, and show that the existence of a Hölder continuous function characterizes qS-reducibility. 


\subsection{Solovay reducibility and Lipschitz continuity}

Definition 4.1 Suppose that $\alpha$ and $\beta$ are real numbers.

(1) $(L)_{1}$ denotes the assertion that there exists a function $f:(-\infty, \beta) \rightarrow(-\infty, \alpha)$ with the following properties.

(a) $f$ is partially computable in the sense of Weihrauch [15].

(b) $f$ is Lipschitz continuous. To be more precise, there exists a positive real number $L$ such that for any $x_{1}, x_{2}<\beta,\left|f\left(x_{1}\right)-f\left(x_{2}\right)\right|<L\left|x_{1}-x_{2}\right|$.

(c) $\{f(x): x<\beta\}$ is cofinal in $(-\infty, \alpha)$. To be more precise, for any $y<\alpha$ there exists $x<\beta$ such that $y \leq f(x)$.

(d) $f$ is nondecreasing.

(2) $(L)_{2}$ denotes the assertion that there exists a function $f:(-\infty, \beta) \rightarrow(-\infty, \alpha)$ that satisfies (a), (b) and (c) above and in addition satisfies

(e) There exists a strictly increasing sequence of rationals $\left\{r_{n}\right\}$ such that $r_{n} \rightarrow \beta-0$ and $f\left(r_{n}\right) \rightarrow \alpha-0$. Here, the sequence $\left\{r_{n}\right\}$ may be non-computable.

Note that $(L)_{2}$ is equivalent to (a) $+(\mathrm{b})+(\mathrm{e})$.

Theorem 4.2 Suppose that $\alpha$ and $\beta$ are left-c.e. reals. Then, the following three assertions are equivalent: $(L)_{1},(L)_{2}$, and " $\alpha \leq_{S} \beta$ ".

Proof $(L)_{1} \Rightarrow(L)_{2}$ is obvious.

$(L)_{2} \Rightarrow \alpha \leq_{S} \beta$ : Assume that $(L)_{2}$ holds with witnesses $f, L$ and $\left\{r_{n}\right\}$. Given a $q \in(-\infty, \beta) \cap \mathbb{Q}$, we are going to choose a rational number $g(q)$. The value $g(q)$ will be an approximate value of $f(q)$. The function $g$ and $L$ will be the witnesses of $\alpha \leq_{S} \beta$.

Let $f(q)=0 . s_{1} s_{2} \cdots$ be a binary expansion where 0 has infinitely many occurrences. Since $f$ is partially computable in the sense of Weihrauch and $q$ is a rational, $f(q)$ is a computable real. In particular, the mapping of $n \mapsto s_{n}$ is a total computable function. For each $n \geq 1$, let $k=k(n)$ be the least $k \geq n$ such that $s_{k}=0$. Then let $c_{n}=0 . s_{1} \cdots s_{k-1} 1$. Each $c_{n}$ is a rational, and $c_{n} \rightarrow f(q)+0$.

Since $\alpha$ is a left-c.e. real, its left set $\{r \in \mathbb{Q}: r<\alpha\}$ is c.e. Therefore, by means of parallel search with respect to $n$, we can effectively find a natural number $n$ such that $c_{n}$ is in the above-mentioned left set. Let $m$ be such a number that we first find. Let $g(q)=c_{m}$. This completes the definition of $g$. 
Now we are going to verify that $g$ and $L$ are the witnesses of the Solovay reducibility. For $n$ that is large enough, it holds that $g(q) \leq f\left(r_{n}\right)$. Thus we have $f(q) \leq c_{m}=g(q) \leq f\left(r_{n}\right)$. Therefore, the following hold for all but finitely many natural numbers $n$.

$$
\left|f\left(r_{n}\right)-g(q)\right| \leq\left|f\left(r_{n}\right)-f(q)\right| \leq L\left|r_{n}-q\right|
$$

The last inequality holds by the Lipschitz continuity of $f$. By taking the limit of $n \rightarrow \infty$, we have $|\alpha-g(q)| \leq L|\beta-q|$. Hence, it holds that $\alpha \leq_{S} \beta$.

$\alpha \leq_{S} \beta \Rightarrow(L)_{1}$ : Suppose $\alpha \leq_{S} \beta$. There exist a partial computable function $f$ and a positive integer $d$ with the following properties. For each rational $q<\beta$, we have $f(q) \downarrow<\alpha$ and $\alpha-f(q)<d(\beta-q)$.

For each point $(w, z)$ such that $w \leq \beta$ and $z \leq \alpha$, we define a closed region $D_{(w, z)}$ as follows.

$$
D_{(w, z)}=\left\{(x, y) \in \mathbb{R}^{2}:-d(w-x)+z \leq y \leq z\right\}
$$

Let $\left\{b_{n}\right\}_{n \in \mathbb{N}}$ be a computable sequence of rationals that increasingly converges to $\beta$. Without loss of generality, we may assume that for each $n$ it holds that $f\left(b_{n}\right)<f\left(b_{n+1}\right)$.

We will prove inductively that there exists a computable subsequence $\left\{b_{n}^{\prime}\right\}_{n \in \mathbb{N}}$ of $\left\{b_{n}\right\}_{n \in \mathbb{N}}$ such that $\left(b_{i}^{\prime}, f\left(b_{i}^{\prime}\right)\right)$ is an interior point of $D_{\left(b_{i+1}^{\prime}, f\left(b_{i+1}^{\prime}\right)\right)}$ for all $i \in \mathbb{N}$. It will be easily seen that $D_{\left(b_{i}^{\prime}, f\left(b_{i}^{\prime}\right)\right)} \subseteq D_{\left(b_{i+1}^{\prime}, f\left(b_{i+1}^{\prime}\right)\right.}$ for all $i \in \mathbb{N}$. Then we will construct the function $g$.

Construction of $\left\{b_{n}^{\prime}\right\}_{n \in \mathbb{N}}$ : Let $b_{0}^{\prime}=b_{0}$. We define $Q_{0}$ as the point $\left(b_{0}, f\left(b_{0}\right)\right)$. By our assumption on $f$ and $d$, it holds that $f\left(b_{0}\right)<\alpha$, and $\alpha-f\left(b_{0}\right)<d\left(\beta-b_{0}\right)$. By means of the last inequality, we have $-d\left(\beta-b_{0}\right)+\alpha<f\left(b_{0}\right)$. Hence $Q_{0}$ is an interior point of $D_{\beta, \alpha}$. Therefore, for any $x \leq b_{0}$ the point $\left(x, f\left(b_{0}\right)\right)$ is an interior point of $D_{(\beta, \alpha)}$.

Now suppose that $n$ is a natural number and we have defined a finite subsequence $b_{i}^{\prime}(i=0, \ldots, n)$ of $\left\{b_{i}\right\}_{i \in \mathbb{N}}$ and suppose that all the points $Q_{i}\left(b_{i}^{\prime}, f\left(b_{i}^{\prime}\right)\right)$ are interior points of $D_{(\beta, \alpha)}$. In Figure 1, the line leading to $(\beta, \alpha)$ denotes the line $y=\alpha, x \leq \beta$. The diagonal line starting from $(\beta, \alpha)$ denotes the line $y=-d(\beta-x)+\alpha$. The region between the two lines is $D_{(\beta, \alpha)}$. The thick half line of Figure 1 is the set $\left\{\left(x, f\left(b_{0}\right)\right): x \leq b_{0}\right\}$.

For sufficiently large $N$, all the points $Q_{i}(i=0, \ldots, n)$ are interior points of the region $D_{\left(b_{N}, f\left(b_{N}\right)\right)}$. We can effectively find one of such $N$. Then $f\left(b_{N}\right)$ is larger than $f\left(b_{n}^{\prime}\right)$, the second coordinate of $Q_{n}$. Let $b_{n+1}^{\prime}$ be $b_{N}$. We define $Q_{n+1}$ as the point $\left(b_{N}, f\left(b_{N}\right)\right)$. Then $Q_{n+1}$ is an interior point of $D_{(\beta, \alpha)}$ in the same manner as $Q_{0}$. 


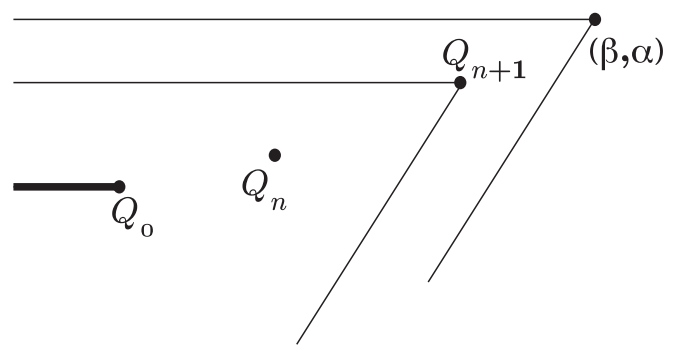

Figure 1: Construction of the graph of $g$

Construction of $g:(-\infty, \beta) \rightarrow(-\infty, \alpha)$ : For each $x \leq b_{0}$ we define $g(x)$ as to be $f\left(b_{0}\right)$. By connecting $Q_{i}$ s we get a line graph, and we define $g(x)$ for $x \leq b_{n}^{\prime}$ by this line graph. This completes the inductive definition of $g:(-\infty, \beta) \rightarrow(-\infty, \alpha)$.

Then $g$ is partially computable in the sense of Weihrauch, and if $x \rightarrow \beta-0$ then $g(x) \rightarrow \alpha-0$. In addition, $g$ is increasing. We are going to show that $g$ is Lipschitz continuous with Lipschitz constant $d$. For each $n$, we denote the $x$-coordinate of $Q_{n}$ by $b_{n}^{\prime}$. Suppose $x_{1}, x_{2}$ are reals such that $x_{1}<x_{2}<\beta$. Let $n$ be the least $n$ such that $x_{2} \leq b_{n}^{\prime}$. In the case of $n=0$ it holds that $\left|g\left(x_{2}\right)-g\left(x_{1}\right)\right|=0 \leq d\left|x_{2}-x_{1}\right|$. Otherwise, we have $n \geq 1$ and the following holds.

$$
\left|\frac{g\left(x_{2}\right)-g\left(x_{1}\right)}{x_{2}-x_{1}}\right| \leq \max \left\{\left|\frac{g\left(b_{j+1}^{\prime}\right)-g\left(b_{j}^{\prime}\right)}{b_{j+1}^{\prime}-b_{j}^{\prime}}\right|: j+1 \leq n\right\}
$$

Recall that for each $j, Q_{j}\left(b_{j}^{\prime}, f\left(b_{j}^{\prime}\right)\right)$ is an interior point of $D_{b_{j+1}^{\prime}, f\left(b_{j+1}^{\prime}\right)}$ and the suffix $\left(b_{j+1}^{\prime}, f\left(b_{j+1}^{\prime}\right)\right)$ of $D$ equals the coordinate of $Q_{j+1}$. Therefore, $b_{j}^{\prime}<b_{j+1}^{\prime}$, and $-d\left(b_{j+1}^{\prime}-b_{j}^{\prime}\right)+g\left(b_{j+1}^{\prime}\right)<g\left(b_{j}^{\prime}\right)<g\left(b_{j+1}^{\prime}\right)$. Therefore, for each $j$, the following holds.

$$
\left|\frac{g\left(b_{j+1}^{\prime}\right)-g\left(b_{j}^{\prime}\right)}{b_{j+1}^{\prime}-b_{j}^{\prime}}\right|<d
$$

Hence, the left-hand side of (4-3) is less than the right-hand side of (4-4). Thus, $\left|g\left(x_{2}\right)-g\left(x_{1}\right)\right| \leq d\left|x_{2}-x_{1}\right|$. Therefore, $(L)_{1}$ holds.

Corollary 4.3 Suppose that $\alpha$ and $\beta$ are left-c.e. reals. Then $\alpha \leq_{S} \beta$ if and only if there exists a rational $s<\beta$ and a function $f:[s, \beta] \rightarrow \mathbb{R}$ with the following properties.

(a) $f$ is partially computable in the sense of Weihrauch.

(b) $f$ is Lipschitz continuous in $[s, \beta]$.

(e) There exists a strict increasing sequence of rationals $\left\{r_{n}\right\}$ such that ( $s \leq r_{n}$, and) $r_{n} \rightarrow \beta-0$ and $f\left(r_{n}\right) \rightarrow \alpha-0$. Here, $\left\{r_{n}\right\}$ may be non-computable. 
Corollary 4.4 Suppose that $\alpha$ and $\beta$ are left-c.e. reals. Then $\alpha \leq_{S} \beta$ if and only if there exists a function $f:(-\infty, \beta) \rightarrow(-\infty, \alpha)$ with the following properties.

(a) $f$ is partially computable in the sense of Weihrauch.

(e) There exists a strict increasing sequence of rationals $\left\{r_{n}\right\}$ such that $r_{n} \rightarrow \beta-0$ and $f\left(r_{n}\right) \rightarrow \alpha-0$. Here, $\left\{r_{n}\right\}$ may be non-computable.

(b') The condition for Lipschitz continuity holds whenever the larger point is some $r_{n}$. More precisely, there exists a positive real number $L$ such that for any $x<\beta$ and any natural number $n$, if $x<r_{n}$ then $\left(f\left(r_{n}\right)-f(x)\right) \leq L\left(r_{n}-x\right)$.

Proof In the proof of Theorem 4.2, the proof of $(L)_{2} \Rightarrow \alpha \leq_{S} \beta$ works in the present setting.

\subsection{QS-reducibility and Hölder continuity}

In this subsection, we are going to extend Corollary 4.4 to the case of qS-reduction and Hölder continuity. As a counterpart to the property (b) in the previous subsection, we investigate the following property $(\mathrm{bH})$.

(bH) $f$ is Hölder continuous with the positive order $<1$. To be more precise, there exists positive real numbers $H$ and $\xi$ such that $\xi<1$ and for any $x_{1}, x_{2}<\beta$, $\left|f\left(x_{1}\right)-f\left(x_{2}\right)\right|<H\left|x_{1}-x_{2}\right|^{\xi}$.

Lemma 4.5 Suppose that $\alpha$ and $\beta$ are left-c.e. reals. Then $\alpha \leq_{q S} \beta$ if and only if there exists a function $f:(-\infty, \beta) \rightarrow(-\infty, \alpha)$ with the following properties.

(a) $f$ is partially computable in the sense of Weihrauch.

(e) There exists a strict increasing sequence of rationals $\left\{r_{n}\right\}$ such that $r_{n} \rightarrow \beta-0$ and $f\left(r_{n}\right) \rightarrow \alpha-0$. Here, $\left\{r_{n}\right\}$ may be non-computable.

(b") The condition for Hölder continuity holds whenever the larger point is some $r_{n}$. More precisely, there exists a positive real number $H$ and a positive integer $\ell$ such that for any $x<\beta$ and any natural number $n$, if $x<r_{n}$ then $\left(f\left(r_{n}\right)-f(x)\right)^{\ell} \leq H\left(r_{n}-x\right)$.

Proof The above assertion implies $\alpha \leq_{q S} \beta$ : The proof is very similar to the counterpart in the proof of Theorem 4.2.

$\alpha \leq_{q S} \beta$ implies the above assertion: Suppose $\alpha \leq_{q S} \beta$. There exist a partial computable function $f$ and positive integers $d$ and $\ell$ with the following properties. For 
each rational $q<\beta, f(q) \downarrow<\alpha$ and we have $(\alpha-f(q))^{\ell}<d(\beta-q)$. We are going to modify the proof of " $\alpha \leq_{S} \beta \Rightarrow(L)_{1}$ " (a part of Theorem 4.2). As before, let $\left\{b_{n}\right\}_{n \in \mathbb{N}}$ be a computable sequence of rationals that increasingly converges to $\beta$, and assume that $f\left(b_{n}\right)<f\left(b_{n+1}\right)$. Recall the roles that region $D_{(w, z)}$ and point $Q_{i}$ played in the proof of Theorem 4.2. We investigate the following new region $E_{w, z}$ in place of $D_{(w, z)}$.

$$
E_{w, z}=\left\{(x, y) \in \mathbb{R}^{2}:-d^{1 / \ell}(w-x)^{1 / \ell}+z \leq y \leq z\right\}
$$

Let $R_{i}(i \in \mathbb{N})$ be the new $Q_{i}(i \in \mathbb{N})$ defined by means of $E_{w, z}$. By connecting $R_{i}$ s we get a line graph, and we define $h(x)$, the counterpart to $g(x)$, by this line graph. For each natural number $i$, let $\left(b_{i}^{*}, f\left(b_{i}^{*}\right)\right)$ be the coordinate of $R_{i}$. By our definition, $b_{0}^{*}=b_{0}$. In Figure 2, the horizontal line leading to $(\beta, \alpha)$ denotes the line $y=\alpha, x \leq \beta$. The curve starting from $(\beta, \alpha)$ denotes the curve $y=-d^{1 / \ell}(\beta-x)^{1 / \ell}+\alpha$. The region between the horizontal line and the curve is $E_{\beta, \alpha}$. The thick half line of Figure 2 is the set $\left\{\left(x, f\left(b_{0}\right)\right): x \leq b_{0}\right\}$.

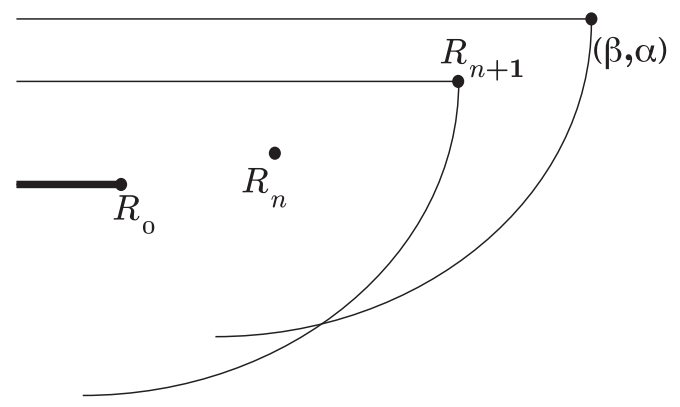

Figure 2: Construction of the graph of $h$

Suppose that $n$ is a natural number and $x$ is a real number such that $x<b_{n}^{*}$. In the case of $n=0$, it holds that $h\left(b_{n}^{*}\right)=h(x)=f\left(b_{0}\right)$. Therefore the inequality $\left(h\left(b_{n}^{*}\right)-h(x)\right)^{\ell} \leq d\left(b_{n}^{*}-x\right)$ is apparent.

If $n>0$ then all of the $R_{i} \mathrm{~s}(i=0, \ldots, n-1)$ are interior points of $E_{b_{n}^{*}, f\left(b_{n}^{*}\right)}$. There are two cases. Case 1: Point $(x, h(x))$ is on the half line " $x \leq b_{0}^{*}$ and $y=f\left(b_{0}^{*}\right)$ ". Case 2: Point $(x, h(x))$ is on line segment $R_{i} R_{i+1}$ for some $i<n$. In the both cases, point $(x, h(x))$ is an interior point of $E_{b_{n}^{*}, f\left(b_{n}^{*}\right)}$. Therefore the inequality $\left(h\left(r_{n}\right)-h(x)\right)^{\ell} \leq d\left(r_{n}-x\right)$ holds.

Now we state and prove the main theorem.

Theorem 4.6 Suppose that $\alpha$ and $\beta$ are left-c.e. real numbers. Then $\alpha \leq_{q S} \beta$ holds if and only if there exists a function $g:(-\infty, \beta) \rightarrow(-\infty, \alpha)$ with the following properties. 
(a) $g$ is partially computable in the sense of Weihrauch.

(b) $g$ is nondecreasing.

(c) If a real $x \rightarrow \beta$ - then $g(x) \rightarrow \alpha-$.

(d) $g$ is Hölder continuous with positive order $<1$.

Proof The "if" direction is immediate from Lemma 4.5. We are going to prove "only if" direction. Suppose that $\alpha \leq_{q S} \beta$. Without loss of generality, we may assume that $\alpha$ and $\beta$ belong to the open interval $(0,1)$. It is enough to construct a function $g:[0, \beta) \rightarrow[0, \alpha)$ with the desired properties; then we extend $g$ to the function from $(-\infty, \beta)$ to $(-\infty, \alpha)$ so that it takes the value $g(0)$ in the interval $(-\infty, 0)$.

Suppose that a partial computable function $f$ and positive integers $d$ and $\ell$ satisfy the following property. For each rational number $q<\beta$ it holds that $f(q) \downarrow<\alpha$ and $(\alpha-f(q))^{\ell}<d(\beta-q)$. In the case where $\ell=1$ the assertion reduces to Theorem 4.2. Throughout the rest of the proof, we assume $\ell \geq 2$. Assume that sequence $\left\{r_{n}\right\}(\nearrow \beta)$ and Weihrauch partial computable function $h$ are those constructed in the proof of Lemma 4.5. We construct a new function $g$ based on $h$.

Let $s=1 / \ell$. By our assumption of $\ell \geq 2$, we have $0<s<1$. We fix a rational number slightly larger than $d^{s}$. Hereafter we let $d$ denote the above-mentioned rational number. For every natural number $n$, the following holds.

$$
\forall k<n\left[h\left(r_{n}\right)-h\left(r_{k}\right)<d\left(r_{n}-r_{k}\right)^{s}\right]
$$

We are going to define a real number $t_{n}$ as a solution of the following equation in variable $x$.

$$
h\left(r_{n}\right)+d\left(x-r_{n}\right)^{s}=h\left(r_{n+1}\right)+d\left(x-r_{n+1}\right)^{s}
$$

The equation (4-7) has a solution $>r_{n+1}$ by the following reason. The equation (4-7) is equivalent to the following.

$$
d\left(x-r_{n}\right)^{s}-d\left(x-r_{n+1}\right)^{s}=h\left(r_{n+1}\right)-h\left(r_{n}\right)
$$

For a while, let $h_{L}(x)$ denote the left-hand side of (4-8). On the one hand, $h_{L}\left(r_{n+1}\right)=$ $d\left(r_{n+1}-r_{n}\right)^{s}$ is, by (4-6), larger than $h\left(r_{n+1}\right)-h\left(r_{n}\right)>0$. On the other hand, $h_{L}(x) \rightarrow 0+$ if $x \rightarrow \infty$, which is verified by means of L'Hospital's rule. Hence, by the Intermediate Value Theorem, (4-7) has a solution $>r_{n+1}$. Let $t_{n}$ be the solution.

In the equation (4-8) $d$ is a rational number, $s$ is $1 / \ell(\ell \in \mathbb{N})$, and function $h(x)$ is computable. Moreover, function $h_{L}(x)$ is strictly decreasing in the interval $x>r_{n+1}$. Therefore we can find the solution $t_{n}$ computably by applying the bisection method. 
We define $A_{n}$ as to be $h\left(r_{n}\right)+d\left(t_{n}-r_{n}\right)^{s}$. Thus $A_{n}=h\left(r_{n+1}\right)+d\left(t_{n}-r_{n+1}\right)^{s}$. By means of $t_{n}$ and $A_{n}$, we define a function $g_{n}$ on the closed interval $\left[r_{n}, r_{n+1}\right]$.

$$
g_{n}(x):=A_{n}-d\left(t_{n}-x\right)^{s}
$$

It is immediate that $g_{n}\left(r_{n+1}\right)=g_{n+1}\left(r_{n+1}\right)$. We define a continuous function $g$ by connecting the graphs of $g_{n}(n \in \mathbb{N})$. It is not hard to see that $t_{n}$ is a computable real number. Therefore we know that $g$ is a partial computable function in the sense of Weihrauch. Thus the assertion (a) of the theorem holds. Of course, $g$ is nondecreasing, and $g(x)$ approaches to $\alpha=\lim _{j \rightarrow \infty} f\left(r_{j}\right)$ when $x \rightarrow \beta$. Thus the assertions (d) and (e) of the theorem hold.

Now we are going to show that $g$ is Hölder continuous with positive order $<1$. Given a positive real number $\varepsilon$ and for each positive real numbers $x, y$ such that $y=x+\varepsilon<1$, we are going to show that $g(y)-g(x)<3 d \varepsilon^{s}$.

Case 1: $x$ and $y$ are in the same interval. To be more precise, $x, y \in\left[r_{n}, r_{n+1}\right)$ for some $n \in \mathbb{N}$.

$$
\begin{aligned}
g(y)-g(x) & =g_{n}(x+\varepsilon)-g_{n}(x) \\
& =\left(A_{n}-d\left(t_{n}-x-\varepsilon\right)^{s}\right)-\left(A_{n}-d\left(t_{n}-x\right)^{s}\right) \\
& =d\left(\left(t_{n}-x\right)^{s}-\left(t_{n}-x-\varepsilon\right)^{s}\right)
\end{aligned}
$$

By means of the inequality $0<s<1$, it is not hard to see that for any real numbers $z$ and $w$, if $0<z<w$ then $w^{s}-z^{s} \leq(w-z)^{s}$. In particular, the last formula of (4-10) is at most $d \varepsilon^{s}$. In summary, it holds that $g(y)-g(x) \leq d \varepsilon^{s}$.

Case 2: otherwise. Then for some $k$ and $n$ such that $k<n$, it holds that $r_{k} \leq x<$ $r_{k+1} \leq r_{n} \leq y<r_{n+1}$. Let $a:=r_{k+1}-x, b:=r_{n}-r_{k+1}$ and $c:=y-r_{n}$.

The inequalities (4-11) and (4-13) reduce to Case 1. Recall that $g$ and $h$ have the same value at an end point of each interval, that is, $g\left(r_{j}\right)=h\left(r_{j}\right)$ for each natural number $j$. Hence the inequality (4-12) reduces to (4-6).

$$
\begin{aligned}
g\left(r_{k+1}\right)-g(x) & <d a^{s} \\
g\left(r_{n}\right)-g\left(r_{k+1}\right) & \leq d b^{s} \\
g(y)-g\left(r_{n}\right) & <d c^{s}
\end{aligned}
$$

Therefore, we have the following.

$$
g(y)-g(x)<d\left(a^{s}+b^{s}+c^{s}\right)
$$

In order to complete Case 2, we are going to employ Hölder's inequality (Beckenbach and Bellman [1]). Under the assumption of $p, q>1$ and $1 / p+1 / q=1$, for any 
nonnegative real numbers $a_{1}, a_{2}, a_{3}, b_{1}, b_{2}$ and $b_{3}$, the following holds.

$$
a_{1} b_{1}+a_{2} b_{2}+a_{3} b_{3} \leq\left(a_{1}^{p}+a_{2}^{p}+a_{3}^{p}\right)^{1 / p}\left(b_{1}^{q}+b_{2}^{q}+b_{3}^{q}\right)^{1 / q}
$$

For our purpose, we investigate the case where $1 / p:=s, 1 / q:=1-s, a_{1}:=a^{s}$, $a_{2}:=b^{s}, a_{3}:=c^{s}$, and $b_{1}=b_{2}=b_{3}:=1$. In this particular setting, the inequality $(4-15)$ is the following.

$$
a^{s}+b^{s}+c^{s} \leq 3^{1-s}(a+b+c)^{s} .
$$

Hence, we have the following.

$$
\begin{aligned}
g(y)-g(x) & <3^{1-s} d(a+b+c)^{s} \\
& =3^{1-s} d \varepsilon^{s}
\end{aligned}
$$

Thus, in the both Cases, we have $g(y)-g(x)<3 d \varepsilon^{s}$. In other words, the assertion (bH) holds. This completes the proof of the theorem.

\section{Acknowledgements}

We are grateful to Hiroyuki Imai, Kazushi Yoshitomi and the anonymous reviewers for their helpful comments. The fourth author was supported by JSPS KAKENHI Grant Number JP16K05255.

\section{References}

[1] E F Beckenbach, R Bellman, Inequalities, Springer, Berlin (1961); https://doi. org/10.1002/bimj.19660080411

[2] C Calude, R J Coles, P H Hertling, B Khoussainov, Degree-theoretic aspects of computably enumerable reals, from: "Models and Computability", (S B Cooper, J K Truss, editors), London Mathematical Society Lecture Note Series 259, Cambridge University Press, Cambridge (1999) 23-39; https://doi.org/10.1017/CBO9780511565670.003

[3] R G Downey, D R Hirschfeld, Algorithmic Randomness and Complexity, Springer, New York (2010); https://doi.org/10.1007/978-0-387-68441-3

[4] A Grzegorczyk, On the definitions of computable real continuous functions, Fund. Math 44 (1957) 61-71; https://doi.org/10.4064/fm-44-1-61-71

[5] K Higuchi, W MP Hudelson, S G Simpson, K Yokoyama, Propagation of partial randomness, Ann. Pure Appl. Logic 165 (2013) 11-12; https://doi.org/10.1016/ j.apal.2013.10.006 
[6] A Kawamura, H Thies, M Ziegler, Average-case polynomial-time computability of Hamiltonian dynamics, from: "43rd International Symposium on Mathematical Foundations of Computer Science, MFCS 2018”, Leibniz International Proceedings in Informatics (2018) 30:1-30:17; https://doi.org/10.4230/LIPIcs.MFCS.2018.30

[7] K-I Ko, H Friedman, Computational complexity of real function, Theoret. Comput. Sci. 20 (1982) 323-352; https://doi.org/10.1016/S0304-3975(82)80003-0

[8] JH Lutz, Effective fractal dimensions, Math. Log. Quart. 51 (2005) 62-72; https://doi.org/10.1002/malq.200310127

[9] E Mayordomo, A Kolmogorov complexity characterization of constructive Hausdorff dimension, Inform. Process. Lett. 84 (2002) 1-3; https://doi.org/10.1016/S00200190(02)00343-5

[10] K Miyabe, A Nies, F Stephan, Randomness and Solovay degrees, Journal of Logic and Analysis 10:3 (2018) 1-13; https://doi.org/10.4115/jla.2018.10.3

[11] A Nies, Computability and Randomness, volume 51 of Oxford Logic Guides, Oxford University Press, Oxford (2009); https://doi.org/10.1093/acprof:oso/9780199230761. 001.0001

[12] R M Solovay, Draft of paper (or series of papers) on Chaitin's work, Unpublished notes (1975) 215 pages

[13] K Tadaki, A generalization of Chaitin's halting probability $\Omega$ and halting self-similar sets, Hokkaido Math. J. 31 (2002) 219-253; https://doi.org/10.14492/hokmj/1350911778

[14] K Tadaki, Partial randomness and dimension of recursively enumerable reals, from: "Proceedings of the 34st International Symposium on Mathematical Foundations of Computer Science (MFCS 2009)", Lecture Notes in Computer Science 5734 (2009) 687-699; https://doi.org/10.1007/978-3-642-03816-7_58

[15] K Weihrauch, Computable analysis: an introduction, Springer, New York (2000); https://doi.org/10.1007/978-3-642-56999-9

Faculty of Liberal Arts, The Open University of Japan, Japan

School of Science and Technology, Meiji University, Japan

Department of Mathematical Sciences, Tokyo Metropolitan University, Japan

Department of Mathematical Sciences, Tokyo Metropolitan University, Japan

kumabe@ouj.ac.jp, research@kenshi.miyabe.name, houji6@gmail.com

(Corresponding author), toshio-suzuki@tmu.ac.jp

Received: 1 May 2019 Revised: 15 April 2020 